\title{
Spectral-fluorescent properties of serum as a reliable marker for early diagnosis of sepsis
}

\author{
Igor Gerych ${ }^{1}$, Olga Bulavenko², Lesya Ostapyuk ${ }^{2}$, * \\ ${ }^{1}$ Surgery Department, Danylo Halytsky Lviv National Medical University, Lviv, Ukraine \\ ${ }^{2}$ Obstetrics and Gynecology Department, Pirogov Vinnytsia National Medical University, Vinnytsia, Ukraine
}

Email address:

lesya_ost@ukr.net (L. Ostapyuk)

\section{To cite this article:}

Igor Gerych, Olga Bulavenko, Lesya Ostapyuk. Spectral-Fluorescent Properties of Serum as a Reliable Marker for Early Diagnosis of Sepsis. Journal of Gynecology and Obstetrics. Vol. 2, No. 5, 2014, pp. 71-74. doi: 10.11648/j.jgo.20140205.11

\begin{abstract}
Poor worldwide outcome of sepsis treatment is related to the lack of effective methods for its rapid diagnosis. We suggest to use the method based on fluorescence spectroscopy studies in vitro and in vivo for diagnosis of sepsis and pre-septic conditions. Obtained by this method results are $24-48$ hours ahead of the data from standard clinical and laboratory tests.
\end{abstract}

Keywords: Sepsis, Purulent-Septic Complications, Method of Fluorescence Spectroscopy

\section{Introduction}

Amongst the leading causes of morbidity and mortality in the world, sepsis occupies one of the dominant positions. In many countries more people are hospitalized each year with sepsis than with a heart attack. Each year this syndrome causes more deaths than prostate cancer, breast cancer, and HIV/AIDS combined. Increasing incidence and mortality of sepsis is observed: the number of sepsis patients hospitalized in the last 10 years has doubled; between one third and one half of sepsis patients dies.

In general, since the introduction of the consensus approach, the current doctrine of adequate decision of the problem of sepsis [9.13] is defined by the two ideas: I. An effective treatment of sepsis is only possible if the diagnosis is made in time; II. The current standard for early diagnosis of sepsis is the verification of at least two criteria of the systemic inflammatory response syndrome (SIRS) and of classical clinical signs of infection focus.

Current diagnostics of sepsis is based on the criteria of the SIRS [14] and on the results of blood tests for sterility $[9,13]$. The "Achilles heel" of the recognized tactics for the sepsis diagnosis is a quite long (2-7 days) waiting period for clinical and laboratory symptoms of an advanced disease and for the results of bacteriological tests. In fact, this waiting period is the time of lost therapeutic options, postponing an adequate treatment, which leads, generally, to unsatisfactory results of sepsis treatment [5].

It should be noted that despite the obvious clinical rationality and progressiveness of the modern doctrine of adequate solving of the sepsis problem, it has some significant disadvantages. They mostly concern the applied problems of the sepsis diagnostics. First, all clinical and laboratory signs of SIRS recommended as early diagnostic criteria for sepsis, form the basis to suspicio sepsis, but not to the confirmed diagnosis, because similar changes in these parameters can be observed not only at sepsis but also at several common inflammatory diseases that are easily treatable and not life threatening. Second, it is not always possible to state the second diagnostic component: the classical clinical signs of the infection focus. Thus, bacteriemia can be detected only in $45-46 \%$ of patients with clinical signs of sepsis. Approximately in $10 \%$ of cases sepsis occurs without formation of a clinically significant primary source of infection.

Thus, a key problem in the sepsis management is its early diagnosis. Reserves of the traditional diagnostic methods here are essentially exhausted; therefore, usage of the luminescent spectral analysis in septicology for early detection of sepsis and of patient conditions with an increased risk of sepsis development could become a new technology that will make a significant progress in sepsis treatment.

In recent decades, the method of fluorescence spectroscopy has been widely and successfully used to diagnose genetic mutations, which lead to an increased 
susceptibility of the human body to certain diseases, including cancer $[10,11,12,15,16]$. These studies open the way for the development of "personal medicine", when each person can determine his/her individual risk for occurrence of certain diseases and take appropriate preventive measures.

Our purpose is to evaluate the efficacy of fluorescence spectroscopy for early diagnosis of sepsis and "pre-septic" states.

\section{Subjects and Methods}

The study design involved the fluorescence spectroscopy of blood serum in different groups of patients (in vivo) and experimental study of the fluorescent spectral characteristics of serum dilutions by bacterial cultures and with distilled water (DW) (in vitro).

The clinical material was taken from 150 patients, divided into three experimental groups. The first (control) group involved 60 people, including 20 healthy people, 20 healthy pregnant women, and 20 women with an uncomplicated postpartum course. The second cohort (comparison group) included 70 patients with "pre-septic" disease and suspected sepsis: 41 patients with surgical "pre-septic" disease and 29 women with postpartum purulent-septic complications. The third (main) group consisted of 20 patients with sepsis.

In general, 150 serum samples were examined by fluorescence spectroscopy with excitation at $280 \mathrm{~nm}$, which corresponds to the luminescence spectrum of human serum albumin [8]. The measurements were performed using the aperture monochromators MDR-2 (St. Petersburg, Russia) and MDR-12 (St. Petersburg, Russia). As a source of the excitation light the deuterium lamp DDS-400 (St. Petersburg, Russia) with a continuous spectrum of radiation in the $\lambda=200-420 \mathrm{~nm}$ range was used. The required wavelength range of the excitation light was isolated by the MDR-12 monochromator. The fluorescence spectra (FS) were analyzed by the MDR-2 monochromator and recorded by the FEU-100 photomultiplier (Moscow, Russia). The signal from the photomultiplier in the single photon registration mode was converted into a digital code by an analog to digital converter (ADC) and subjected to computer processing. Experimental results were presented in graphical and digital forms. Fluorescence excitation spectra (FES) of biological fluids were measured using the same setup.

In the in vitro studies the FS of 11 dilutions of serum by non-centrifuged (NCF) and centrifuged (CF) cultures of bacteria [6] were measured for two similar experimental series of dilutions with concentrations starting from $100 \%$ of the standard serum down to the pure bacterial cultures (NCF/CF, respectively) with an experimental step of $10 \%$ dilution. In the in vitro studies the FS of 11 dilutions of serum with DW also measured [6]. Excitation of these solutions was also performed at $280 \mathrm{~nm}$.

\section{Results}

In healthy individuals of the first group, the obtained serum FS was the $\lambda$-type curve between 300 and $450 \mathrm{~nm}$ with a maximum (intensity peak) at $340 \mathrm{~nm}$. The fluorescence intensity of blood serum (BS) taken from pregnant women was slightly higher than of non-pregnant women, probably due to lower levels of total protein, mainly albumin, as compared to those of the healthy control group and women with an uncomplicated postpartum course.

For the second group of patients with the "pre-septic" pathology and suspected sepsis, the FS peak intensity at $340 \mathrm{~nm}$ decreased, and a smooth long-wavelength shift (fig.1, curves 2-4) was gradually formed with a tendency to appearance of a new pathological "septic" peak at $380 \mathrm{~nm}$ at deterioration of patients condition .

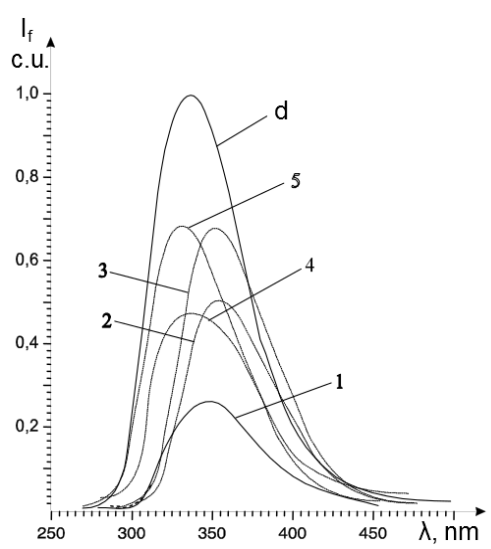

Fig. 1. Fluorescence spectra (FS) of blood serum of patients with the "pre-septic" pathology.

For patients with sepsis (the third group) two peaks in FS (the "septic" peak at $380 \mathrm{~nm}$ and a small "normal" one in the region of $340 \mathrm{~nm}-$ fig. 2, curve 1) were revealed. In the process of patients treatment, the peak at $380 \mathrm{~nm}$ gradually disappeared, whereas the intensity of the peak at $340 \mathrm{~nm}$ gradually increased and became the same as in the healthy control group (fig. 2, curves 2-4).

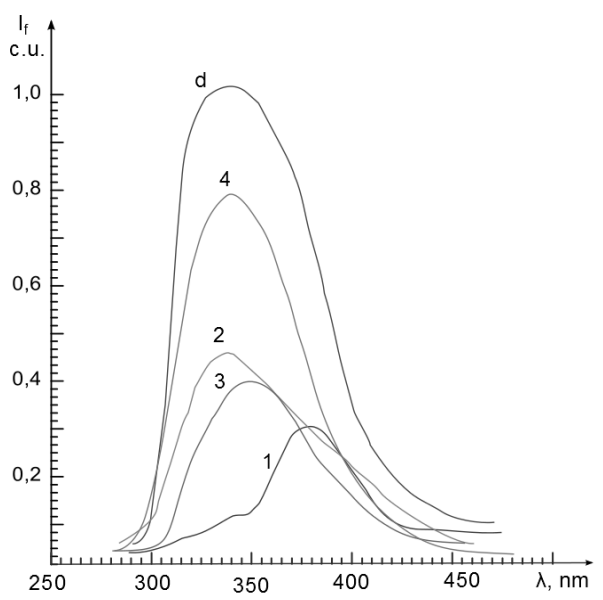

Fig. 2. Fluorescence spectra of blood serum of septic patient $(340 \mathrm{~nm}-$ «normal peak», 380nm-《 septic peak»). 
The pathological two-peak structure of the FS at sepsis is associated with the existence of two types of molecules of albumin: normal albumin (to which the peak in the region of $340 \mathrm{~nm}$ corresponds) and albumin bound by metabolic products of bacteria (the peak in the region of $380 \mathrm{~nm}$ ). Normally, the peak at $380 \mathrm{~nm}$ is absent. But the syndrome of endogenous intoxication creates the conditions for the formation of albumin forms with altered physical and chemical properties [1]. Since albumin has the ability to form complexes, some of its molecules bind toxic metabolic products of bacteria. These albumin molecules become blocked by toxins, therefore, the albumin capacity to bind physiological ligands and drugs decreases [4]. Thus, though the total concentration of albumin is constant, its "effective" concentration decreases [4]. The authors formulated the basic laws of behavior of spectral fluorescence characteristics of BS at purulent-septic diseases that are accompanied by endogenous intoxication, including their dynamics during treatment. A high efficiency of fluorescence spectroscopy for early diagnosis of sepsis is demonstrated.

In vitro studies have established that the fluorescence intensity of NCF and $\mathrm{CF}$ serum dilutions gradually decreases with increasing content of bacterial culture in the solution. We also observe a long-wavelength shift of the fluorescence bands $(\lambda \max )$ of dilutions with the pathological tendency to "septic" peak in the long-wave region (fig.3) [2]. The observed changes in the spectral fluorescent characteristics of the sepsis patients serum and its NCF and CF dilutions are caused by bacteria and their metabolic products.

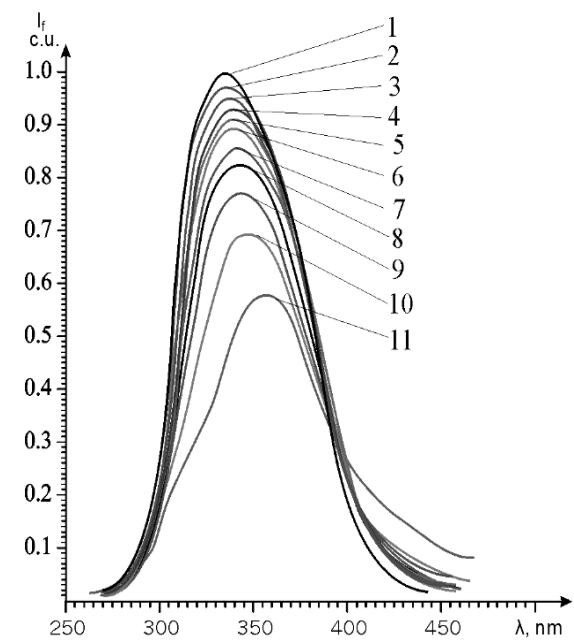

Fig. 3. Effect of dilution centrifuged $(C F)$ crops on fluorescence spectra of donor blood serum (BS) (1-blood serum (BS) 2 - 90\% BS 3 - 80\% BS, 4 $70 \%$ BS, 5 - 60\% BS, 6 - 50\% BS, 7 - 40\% BS, 8 - 30\% BS, 9 - 20\% BS, 10 - $10 \%$ BS, $11-C F$ crops).

Fluorescent spectral characteristics of $20 \%$ donor albumin are very close to those of BS, which allows one to use it as a reference in our studies of the FS of BS (Figure 4) [2]. In our in vitro experiments we explored the FS of 11 dilutions of BS with DW. The results are presented in Figure 5. An enhancement of BS fluorescence with increasing DW content is observed. Thus, a gradual decrease in the number of albumin molecules in the BS reduces the concentrational quenching of BS fluorescence. These results are specific and allow one to study the diseases accompanied by hypoproteinemia and hypoalbuminemia, as well as to explore the influence of different therapeutic measures (to study the effect of fluid therapy on the human body).

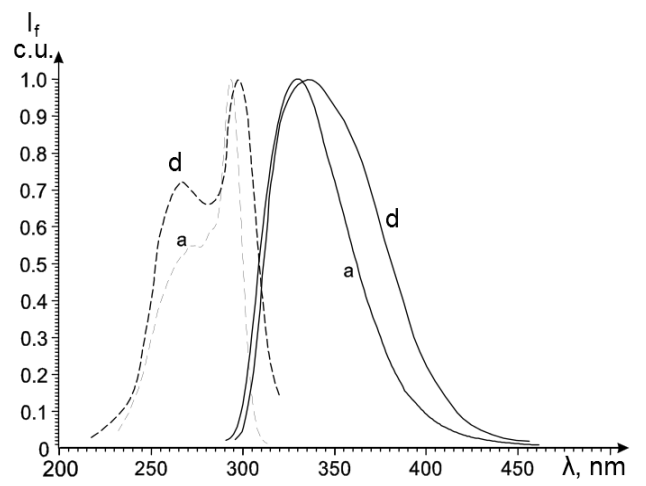

Fig. 4. Fluorescence excitation spectra bands $(\lambda \max =340 \mathrm{~nm}(d, a))$ and fluorescence spectra $(\lambda$ exc. $=280 \mathrm{~nm}(d, a))$ serum donor $(d)$ and $20 \%$ albumin solution (a).

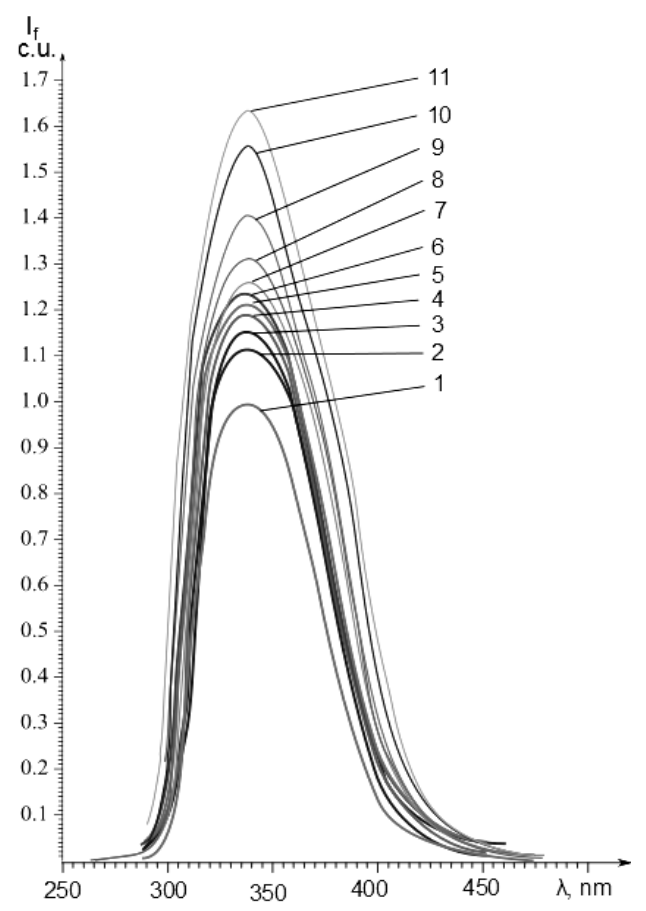

Fig. 5. Effect of dilution with distilled water $(D W)$ on the fluorescence spectra of donor blood serum (BS)(1 - BS 2 - 90\% BS, 3 - 80\% BS, 4 - 70\% $B S, 5-60 \% B S, 6-50 \% B S, 7-40 \% B S, \quad 8-30 \% B S, 20 \% B S, 10-10 \% B S$, $\left.11-5 \% B S, 12-D W: I_{f}=0\right)$.

It has already been noted that in our studies the fluorescence intensity of BS taken from healthy pregnant women was slightly higher than for normal donors. These results can be explained by the physiological changes in their bodies during pregnancy. It is known that blood volume of pregnant women increases, whereas the protein content is not changed. Thus, they usually have relative hypoproteinemia. It should be noted that measurements of 
FS of BS taken from healthy pregnant women can be considered as a particular case of the similar measurements of BS dilutions with DW (from the studies of "the in vitro diseases models") [2]. Thus, the results of our clinical studies are in a good agreement with the in vitro experimental data.

Thus, the results of in vitro and in vivo studies give reason to consider the fluorescence spectroscopy as an effective method for early diagnosis of sepsis and conditions with an increased risk of its development. Appearance of the "septic" peak at $380 \mathrm{~nm}$ is a reliable marker of sepsis, whereas the peak intensity redistribution (the $380 \mathrm{~nm}$ peak lowers down and the $340 \mathrm{~nm}$ grows up) is indicative of a positive dynamics of the disease.

Spectral-fluorescent properties of serum are a reliable marker for early diagnosis of sepsis, particularly in surgical and obstetric practice. The study of these parameters in dynamics allows one to get a reliable prognosis of the decease, as the changes in the FS appear 24-48 hours ahead of the standard laboratory and clinical parameters. Fluorescence spectroscopy complements information obtained from these tests in terms of the in-depth information about the processes that occur in humans suffering from inflammatory diseases and gives the possibility for prognostic evaluation.

Application of fluorescence spectroscopy in medicine opens new possibilities for the study of physiological and pathological processes patterns of the human body. Spectral-fluorescent properties of serum can be considered as an effective method for early diagnosis of sepsis and its clinical course, as well as verification of the diseases with an increased risk of the sepsis development.

\section{Acknowledgements}

The authors are grateful to professors Anatolii Voloshinovskii, Stepan Myagkota and Roman Levitskii for valuable advice and guidance in the preparation of this publication.

\section{References}

[1] Ya. Hruzunov and G.E. Dobretsov Albumin of blood serum in clinical medicine; Moscow: HEOTAR, 1998, 440.

[2] O.V. Bulavenko, I.D. Gerych, L.R. Ostapyuk, A.S. Voloshinovskii, S.V. Myagkota and V.V. Vashcuk Modeling changes in blood serum in various diseases and medical events; Biomedical and biosocial anthropology, Vinnytsia , 2013; 20 February: 8-14.

[3] O.V. Bulavenko and L.R. Ostapyuk Urgent postnatal diagnosis of septic complications in modern obstetrics; Journal of Morphology. Reports of Morphology, Vinnytsia, 2011; 17 (3 October): 666-669.

[4] V. Gavrylov, M.Bydula and D.Furmanchuk Reduction effective concentrations of albumin as a marker between accumulation and binding toxic products in plasma during endogenous intoxication, in Albumin of blood serum in clinical medicine, Ya. Hruzunov and G.E. Dobretsov, Eds. Moscow: HEOTAR, 1998, pp. 132-139.

[5] I. Gerych, L. Levytska, A.Voloshinovskii and S.Myagkota Fluorescent analysis as a method for diagnosis of sepsis; Bullet. of Lviv. University. Biology Series, Issue 32. Lviv: Lviv Ivan Franko Univ., 2003; pp. 23-30.

[6] I.D. Gerych, L.R. Levytska, A.S.Voloshinovskii and S.V. Myagkota Fluorescent spectral model of sepsis in vitro; Math sciences. conf. Current issues and abdominal septic surgery. Lviv, 2004; pp.111-112.

[7] I.D.Gerych,L.R.Ostapyuk, V.V.Vashcuk, A. S.Voloshinovskii and S.V. Myagkota Prospects for diagnosis of sepsis and septic complications by fluorescence spectroscopy; Bullet. Ukr. med. stomat. Acad. Actual problems of modern medicine, Poltava, 2009; 9, 1 (25); pp. 248-256.

[8] Pat. Ukraine № 76953 A61V 17/00 G01N 33/48, G01N $21 / 64$ method for early diagnosis of septic complications by the method of fluorescence spectroscopy / I.D. Gerych, O.V. Bulavenko, L.R.Ostapyuk, A.S.Voloshinovskii, S.V. Myagkota applicant and patentee Vinnytsia National Medical University.-№ 201207441,appl.19.06.2012; publ. 25.01.2013, Bull. № 2.

[9] P. Y. Bochud, M.P. Glauser and T. Calandra Antibiotics in sepsis; Intensive Care Med., 2001; 27; pp. 33-48.

[10] J. Gabert, E. Beillard, Velden van der VHJ, Bi W, D. Grimwade, N. Pallisgaard, G. Barbany, G. Cazzaniga, J.M. Cayuela, H. Cave, F. Pane, JLE Aerts, D Dei Michel, X Thirion, V. Pradel, M. Gonzalez, S. Viehmann, M. Malec, G. Saglio and Dongen van JJM Standardization and quality control studies of «real-time» quantitative reverse transcriptase polymerase chain reaction of fusion gene transcripts for residual disease detection in leukemia; A Europe Against Cancer Program. Leukemia, Paris, 2003; 17 ; pp. 2318-2357.

[11] M. Hafes, Ye F., K. Jackson, Z. Yang, J. E. Karp, E. Labourier, and C. D. Gocke Performance and clinical evaluation of a sensitive multiplex assay for the rapid detection of common NPM1 mutations; Journal of Molecular diagnostics, Paris, 2010; 12 (5) September ; pp. 629-635.

[12] E. Hammond, K. Shaw, B. Carnley, S. P'ng, I. James and R. Herrmann Quantitative determination of JAK2 V617F by TaqMan. An absolute measure of averaged copies per cell that may be associated with the different types of myeloproliferative disorders; Journal of Molecular diagnostics, Paris, 2007; 9 (2) April ; pp. 242-248.

[13] M. Llewelyn and J. Cohen Diagnosis of infection in sepsis; Intensive Care Med., $2001 ; 27$; pp. 10-32.

[14] I. Matot and C.L. Sprung Definition of sepsis; Intensive Care Med., 2001; 2; pp. 3-9.

[15] M. Nauck, W. Marz, and H. Wieland Evaluation of the Roche Diagnostics LightCycler-Factor V Leiden mutation detection kit and the LightCycler-Prothrombin mutation detection kit; Clinical biochemistry, 2000; 33 (3); pp. 213-216.

[16] E. C. Wolstencroft, K. Hanlon, L. W. Harries, G. R. Standen, A. Sternberg and S. Ellard Development of quantitative real-time polymerase-chain reaction assay for the detection of the of JAK2 V617F mutation disorders; Journal of Molecular diagnostics, 2007; 9 (1); pp. 42-46. 